\title{
Eine Welt im Werden
}

\author{
Fritz Bopp \\ Z. Naturforsch. 40a, 1171-1181 (1985); received August 19, 1985 \\ The World is Steadily Coming to Be \\ If $\mu$ equals the average value of the cosmical density of matter, and if $G$ equals Newton's \\ gravitational constant, the length $R=c / \sqrt{G \mu}$ nearly yields the radius of the world. Therefore it \\ should not be necessary to introduce a second radius $K$ of the same kind as in Einstein's or \\ Friedman's line element $\mathrm{d} s^{2}=-g_{\mu v}(x / K) \mathrm{d} x^{\mu} \mathrm{d} x^{v}$. For this reason, we apply Einstein's field \\ equations on the line element $\mathrm{d} s^{2}=\mathrm{d} t^{2}-f(\varrho)^{2} \mathrm{~d} r^{2}, \varrho=r / t, c=1$, and obtain a world which is \\ steadily coming to be. The Big-Bang-world is replaced by an expanding one whose mass $M$ is \\ steadily growing according to $\mathrm{d} M / \mathrm{d} t \sim c^{3} / G$. It should be taken into account that less \\ assumptions are necessary for a general relativistic world which is coming to be.
}

\section{$\S 1$. Motivation}

Die hier untersuchte „Welt im Werden“ ist wie die Friedman-Welt [1] ein Modell des Kosmos, das aus einer strengen Lösung der Einsteinschen Feldgleichungen [2] hervorgeht. Da die Friedman-Welt mathematisch-ästhetisch sehr befriedigend ist, muß man sagen, warum eine alternative Lösung beachtenswert erscheint. Wir behaupten, daß die Welt im Werden eher mit der puritanischen Wissenschaftslehre von Newton [3] im Einklang ist als die Friedmansche.

Im Zentrum der Newtonschen Wissenschaftslehre steht seine Definition des Begriffs Axiom: „Axiome gehen aus Sätzen hervor, die aus Phänomenen abgeleitet und die dann durch Induktion allgemeingültig gemacht werden." Wie wir an anderer Stelle ausgeführt haben [4], sind Axiome Newtonscher Art innerhalb des Geltungsbereiches phänomenologisch begründeter Sätze widerspruchsfrei, weil das, was man wirklich tun kann, logisch nicht anfechtbar ist. Axiome der oben definierten Art brauchen aber nicht ewig zu gelten, weil neue Phänomene auftauchen können, die andere und insbesondere umfassendere Axiome erfordern. Sie müssen der harten Bedingung genügen, die alten und die neuen Phänomene zu umschließen. Das garantiert trotz sich wandelnder Bilder ein stetes Fortschreiten der Erkenntnis.

Obige Definition macht sofort verständlich, warum Einsteins ursprüngliche Fassung sich gegen

Sonderdruckanforderungen an Prof. Dr. F. Bopp, Sulzbacher Str. 3, D-8000 München 40. alle späteren Erweiterungen der Theorie durchgesetzt hat. Sie basiert auf einem vorher unbeachtet gebliebenen Phänomen, nämlich dem der lokalen Äquivalenz von Trägheit und Schwere, und ist darum frei von Hypothesen im Sinne von Newton (l.c. [3]) : ,Was immer nicht aus Phänomenen abgeleitet ist, muß Hypothese heißen; Hypothesen, seien es metaphysische oder physikalische, seien es okkulte oder mechanische Eigenschaften, haben in der Experimentalphilosophie keinen Platz.“

Das ist's, warum Newtons Wissenschaftslehre puritanisch genannt werden kann. Er verwirft damit willkürliche Spekulationen. Die Mannigfaltigkeit des Denkbaren ist nämlich so gewaltig, daß man mit willkürlichen Axiomen zwar intellektuell höchst befriedigende Theorien entwickeln, aber kaum hoffen kann, die der Welt innewohnende, vom Menschen nach und nach zu erkennende, niemals manipulierbare Ordnung in den Griff zu kriegen.

Längst vor der Entdeckung der Relativitätstheorien Einsteins war eines der kosmischen Basisphänomene bekannt. Wäre der Raum gleichmäßig mit Sternen erfüllt, wäre die mittlere Leuchtkraft der Sterne endlich, gäbe es ferner keine interstellare Absorption des Lichtes, so würde zwar die Helligkeit der einzelnen Sterne mit dem reziproken Quadrat des Abstands ab-, die Anzahl der mit einem Blick erfaßten Sterne aber mit dem Quadrat zunehmen, so daß der Nachthimmel gleichmäßig hell wäre etwa wie die Sonnenscheibe.

Man hat schon früh daraus geschlossen, daß die von uns wahrnehmbare Welt endlich sein müsse. Sei $\mu=10^{-25} \mathrm{~kg} \mathrm{~m}^{-3}$ die mittlere Massendichte im Kosmos, spielt die Gravitationskonstante $G=6,67$

0340-4811/85/1200-1171\$01.30/0. - Please order a reprint rather than making your own copy. 
$\cdot 10^{-11} \mathrm{~kg}^{-1} \mathrm{~m}^{3} \mathrm{~s}^{-2}$ eine für die Ausdehnung der Welt wesentliche Rolle, hinter der andere Ursachen quantitativ zurücktreten, so erhält man für den Weltradius allein aus Dimensionsgründen etwa die Größe

$$
R=\frac{c}{\sqrt{G \mu}}=1,16 \cdot 10^{26} \mathrm{~m} .
$$

Dieser Radius ist mindestens größenordnungsmäßig mit den Radien vereinbar, zu denen die mittleren Lebensdauern der natürlich radioaktiven Atomkerne führen.

Ein zweites kosmisches Basisphänomen hat Hubble entdeckt [5]. Es ist die Rotverschiebung der Spektren ferner Nebel gemäß

$$
\frac{\Delta v}{v}=H \cdot d, \quad H=0,86 \cdot 10^{-26} \mathrm{~m}^{-1}
$$

mit wachsendem Abstand $d$. $H$ ist die HubbleKonstante. Diese Entdeckung ist erst erfolgt, nachdem vor allem Einstein und Friedman allgemein relativistische Weltmodelle angegeben hatten. Doch ist Hubbles Entdeckung unabhängig von Theorien erfolgt. Es ist ein Basisphänomen, das unabhängig von speziellen Modellen Bestand hat.

Auch die Hubble-Konstante ist mit obigem Weltradius verträglich. Denn gemäß

$$
H R=\frac{c H}{\sqrt{G \mu}}=0,9990
$$

sind $R$ und $H^{-1}$ von gleicher Größenordnung. Ein Wert so nahe bei 1 mag zufällig sein. Insbesondere könnte die Gleichung für $R$ und $G$ bei voll formulierter Theorie noch einen Zahlenfaktor enthalten.

Einstein hat früh versucht [6], ein statisches Weltmodell allgemein relativistisch zu formulieren und sich dabei von einer vorrelativistischen Idee von Seeliger [7] leiten lassen. Ein solches Modell existiert, wenn man zu den ursprünglichen Feldgleichungen Einsteins ein kosmologisches Glied hinzufügt, von dem Einstein selbst später drastisch abgerückt ist. Bereits 1922 konnte Friedman zeigen [8], daß man ohne Änderung der Einsteinschen Feldgleichungen $\mathrm{zu}$ einem endlichen Kosmos gelangt, wenn man zuläßt, daß sich der Weltradius mit der Zeit ändert. Verständlicherweise hat seine Theorie erst nach der Entdeckung des Hubble-Effektes Beachtung gefunden. Doch ist das Linienelement seiner Theorie im Sinne von Newton nicht frei von spekulativen Elementen.
Gewöhnlich schreibt man allgemein relativistische Linienelemente in der Form

$$
\mathrm{d} s^{2}=-g_{\mu v}(x) \mathrm{d} x^{\mu} \mathrm{d} x^{v} .
$$

Darin können $\mathrm{d} x$ und $\mathrm{d} s$ die Dimension $m$ haben. Der metrische Tensor ist dann dimensionlos. Läßt man beliebige Funktionen zu, so muß aus Dimensionsgründen $x$ durch $x / K$ ersetzt werden, worin $K$ eine Längenkonstante von der Größe des Weltradius ist. Darum lautet das dimensionsrichtig geschriebene Linienelement

$$
\mathrm{d} s^{2}=-g_{\mu v}(x / K) \mathrm{d} x^{\mu} \mathrm{d} x^{v} .
$$

Bei Koordinaten, die wie Polarkoordinaten dimensionslos sind, gilt obiges sinngemäß für etwaige Faktoren vor $\mathrm{d} \vartheta^{2}, \mathrm{~d} \varphi^{2} \mathrm{u}$. dgl.

Da eine solche Längenkonstante bereits im Wechselwirkungsterm der Einsteinschen Feldgleichungen vorkommt und durch die Gravitationskonstante $G$ bestimmt ist, werden zwei unabhängige Weltradien eingeführt, die Konkurrenzsituationen ergeben können. In der Friedmanschen Theorie erhält man darum zwei verschiedene Typen von Modellen, das elliptische für hinreichend große Massendichten und das hyperbolische für kleine. So lange die zweite Längenkonstante nicht zwingend aus Phänomenen hervorgeht, muß man sie nach Newton als unzulässige Hypothese betrachten. Man sollte das Linienelement so formulieren, daß die Konstante $K$ herausfällt. Vielleicht ist die Vermutung, daß die Masse der Dunkelmaterie des Kosmos 10- oder $100 \mathrm{mal}$ so groß sein soll als die der leuchtenden, nur eine Folge der Hypothese. Hier ist es qualitativ unwesentlich, ob die Dichte der Dunkelmaterie klein oder groß ist.

\section{§ 2. Das hypothesenfreie Linienelement}

Die Dimensionskonstante $K$ wird entbehrlich, wenn in den Argumenten von $g_{\mu v}$ nur die Verhältnisse $x^{0}: x^{1}: x^{2}: x^{3}$ vorkommen, wenn also mit $c=1$

$$
g_{\mu v} \equiv g_{\mu v}(\varrho), \quad \varrho=r / t
$$

ist. An anderer Stelle ist gezeigt [9], daß die Eigenzeit nur dann mit Borduhren meßbar ist, wenn für $\mathrm{d} \boldsymbol{r} / \mathrm{d} t=0$ die Gleichung $\mathrm{d} s=\mathrm{d} t$ gilt. Bechränkt man sich außerdem wie Friedman zunächst auf kugelsymmetrische Systeme, so erhält man schließ- 
lich das Linienelement

$$
\mathrm{d} s^{2}=\mathrm{d} t^{2}-f(\varrho)^{2} \mathrm{~d} \boldsymbol{r}^{2}, \quad \varrho=r / t, \quad c=1 .
$$

Die Metrik ist nicht homogen; doch ist nach (5.10) die Dichte $\mu_{0}=$ const.

In der Nähe des Zentrums kann sich die Gravitation wie klassisch physikalisch im Zentrum einer Massenkugel nicht auswirken. Die Metrik sollte darum in $\varrho=0$ Minkowskisch sein. Das führt zu den Randbedingungen

$$
f(\varrho)=e^{u(\varrho)}, \quad u(0)=0, \quad u^{\prime}(0)=0 .
$$

Es wird sich zeigen, daß $u^{\prime}$ und alle höheren $A b-$ leitungen von $u$ in $\varrho=0$ verschwinden, wenn $u(0)=0$ ist. Darum enthält (2.3) nur eine unabhängige Randbedingung. Eine zweite kann z.B. asymptotisch gefordert werden.

Als spezielle Folge der Einsteinschen Feldgleichungen wird sich eine Differentialgleichung zweiter Ordnung ergeben. Das asymptotische Verhalten der Lösungen legt

$$
f(\varrho) \rightarrow 0, u(\varrho) \rightarrow-\infty \text { für } \varrho \rightarrow \infty
$$

als zweite Randbedingung nahe. Tatsächlich wird $f(\varrho)$ so rasch nach 0 gehen, daß z. B. das Volumenintegral gemäß

$$
\tau=\int \sqrt{g} \mathrm{~d} \tau=4 \pi t^{3} \int_{0}^{\infty} f(\varrho)^{3} \varrho^{2} \mathrm{~d} \varrho
$$

endlich bleibt. Dasselbe gilt für den Kugelradius

$$
R=\int_{0}^{\infty} \sqrt{g_{\sigma \sigma}} \mathrm{d} r=t \int_{0}^{\infty} f(\varrho) \mathrm{d} \varrho \text {. }
$$

Klarerweise wachsen $R$ und $\tau$ proportional $\mathrm{zu} t$ bzw. $t^{3}$.

Es besteht eine gewisse Verwandtschaft zur Friedman-Welt. Der Abstand zweier beliebiger Punkte auf einer Kugelfläche $\varrho=$ const. verschwindet im Limes $\varrho \rightarrow \infty$. Die unendlich ferne Kugelfläche ist also in Wahrheit ein Punkt. In diesem Sinne ist auch die durch (2.2) definierte Welt wie die Friedmansche eine endliche.

$\mathrm{Da}$ es in (2.2) keine Konstante von Längendimension gibt, ist die Masse $M$ der Gesamtwelt aus Dimensionsgründen bis auf einen Zahlenfaktor $\gamma$ gleich

$$
M=\gamma \frac{c^{3} t}{G}
$$

Sie nimmt also proportional zur Zeit zu. Darum sprechen wir von einer Welt im Werden. Durch Ver- gleichung mit der Protonenmasse erhält man als Erzeugungsrate

$$
\frac{1}{m_{\mathrm{p}}} \cdot \frac{\mathrm{d} M}{\mathrm{~d} t}=2,424 \cdot 10^{62} \gamma \mathrm{s}^{-1} .
$$

Die Massendichte ist von der Größe

$$
\mu=\frac{3 \gamma}{4 \pi} \frac{1}{G t^{2}} .
$$

Sie ist zwar für $t=0$ singulär, aber um riesige Zehnerpotenzen weniger als bei Friedman. Nach den berühmten drei Minuten von Weinberg [10] ist $\mu$ für $\gamma=1$ rund $110 \mathrm{~g} / \mathrm{cm}^{3}$ also nur etwa $100 \mathrm{mal}$ größer als die von Wasser.

Ganz unabhängig davon, wie man am Ende die Welt im Werden physikalisch beurteilen wird, zeigt sich, mit welcher Unsicherheit Extrapolationen bis zu $t=0$ behaftet sind. Die Vertrautheit mit der Friedman-Welt wird den Zugang zu Alternativen erschweren. Doch muß man zugeben, daß die strenge Anlehnung an Newtons puritanische Lehre eher Vertrauen verdient als durch Gewohnheit Vertrautes.

Da nach (2.8) die Dichte zur Zeit $t=0$ immer noch unendlich wird, liegt die Vermutung nahe, daß man zur Entscheidung zwischen den Alternativen die Quantenphysik der Gravitation braucht. Doch dürfte der Weg bis zur quantenphysikalischen Berechnung der Produktionsrate trotz den ermutigenden Ansätzen von Schäfer und Dehnen [11] noch weit sein. In Hinblick auf die Quantenphysik wird man jedoch zweierlei sagen dürfen. Erstens folgt daraus, daß Teilchenerzeugungen wie -vernichtungen lokale Ereignisse [12] sind: Die Quellen für die Massenzunahme müssen quantenphysikalisch in Teilchen verankert sein. Zweitens muß es sich um einen Effekt handeln, der allein in den Massen der Teilchen bzw. in ihrem Gravitationsfeld verankert ist. Es muß belanglos sein, ob die Erzeugung von Protonen, Elektronen oder anderen Teilchen ausgeht.

Bekanntlich ist das Verhältnis der elektrischen potentiellen Energie zu der der Gravitation zwischen Proton und Elektron gleich

$$
e^{2} / 4 \pi \varepsilon_{0} r: G m_{\mathrm{p}} m_{\mathrm{e}} / r=2,27 \cdot 10^{39} .
$$

Da diese geheimnisvolle Zahl bei vielen, physikalich unabhängig erscheinenden, aber empirisch bestimmten Verhältnissen auftritt, möchte man sie 
rational verstehen. Nach Dirac [13] kann man das erhoffen, wenn sie sich mit der Zahl der Nukleonen in der Welt in Verbindung bringen läßt. Hier hat sich gezeigt, daß dies in der Welt im Werden ohne Hypothesen möglich ist.

\section{§ 3. Energie-Impulstensor}

Bei gegebenem Linienelement folgt der EnergieImpulstensor aus den Einsteinschen Feldgleichungen [14]

$R_{\mu v}-\frac{1}{2} R g_{\mu v}=\varkappa T_{\mu v}, \quad R=g^{\varrho \sigma} R_{\varrho \sigma}, \quad \varkappa=\frac{8 \pi G}{c^{4}}$.

Die Dimensionen von $R_{\mu v}, \varkappa$ und $T_{\mu v}$ sind $\mathrm{m}^{-2}$, $\mathrm{kg}^{-1} \mathrm{~m}^{-1} \mathrm{~s}^{2}$ bzw. $\mathrm{kg} \mathrm{m}^{-1} \mathrm{~s}^{-2}$ oder, wenn $c=1$ ist, $\mathrm{m}^{-2}, \mathrm{~kg}^{-1} \mathrm{~m}$ bzw. $\mathrm{kg} \mathrm{m}^{-3}$. Darin ist

$R_{\mu v}=\partial_{\varrho} \Gamma_{\mu v}^{\varrho}-\partial_{v} \Gamma_{\varrho}^{\varrho}+\Gamma_{\varrho}^{\varrho} \dot{\rho} \Gamma_{\mu v}^{\dot{\partial}}-\Gamma_{\sigma \mu}^{\varrho} \Gamma_{\varrho v}^{\sigma}$

und

$\Gamma_{\mu \nu}^{\varrho}=g^{\varrho \sigma} \Gamma_{\sigma \mu v} ; \quad \Gamma_{\sigma \mu v}=\frac{1}{2}\left(\partial_{\mu} g_{v \sigma}+\partial_{v} g_{\mu \sigma}-\partial_{\sigma} g_{\mu v}\right)$.

Da $g_{\mu v}$ nur von $\varrho=r / t$ abhängt, und da jede Ableitung von $\varrho$ gemäß

$$
\partial_{0} \varrho=-\frac{1}{t} \varrho, \quad \nabla \varrho=\frac{1}{t} \boldsymbol{n}, \quad \boldsymbol{n} \equiv \boldsymbol{r} / r,
$$

einen Faktor $1 / t$ erzeugt, hängen $t \Gamma_{\mu \nu}^{o}$ und $t^{2} R_{\mu v}$ nur von $\varrho$ ab. Danach kann man wegen der sphärischen Symmetrie des Linienelements

$$
\begin{aligned}
& t^{2} R_{00}=A(\varrho), \quad t^{2} R_{0 i}=B(\varrho) n_{i}, \\
& t^{2} R_{i k}=C(\varrho) \delta_{i k}+D(\varrho) n_{i} n_{k}
\end{aligned}
$$

schreiben. Es genügt also $R_{00}, R_{01}, R_{12}$ und $R_{11}$ zu berechnen. Wegen

$$
\begin{aligned}
& \left(g_{\mu v}\right)=\operatorname{diag}\left(-1, f^{2}, f^{2}, f^{2}\right), \\
& g^{2}=-\operatorname{det}\left(g_{\mu v}\right)=f^{6}
\end{aligned}
$$

ist der Krümmungsskalar durch

$$
t^{2} R=-A+\frac{1}{f^{2}}(3 C+D)
$$

bestimmt.

Nur folgende Ableitungen von $g_{\mu \nu}$ sind von 0 verschieden

$$
\begin{aligned}
& t \partial_{0} g_{i i}=-2 \varrho f f^{\prime}=-2 \varrho f^{2} u^{\prime}, \\
& t \partial_{k} g_{i i}=2 f f^{\prime} n_{k}=2 f^{2} u^{\prime} n_{k} .
\end{aligned}
$$

Damit erhält man aus (3.3) für $i \neq k$

$t \Gamma_{i i}^{0}=-\varrho f^{2} u^{\prime}, \quad t \Gamma_{i i}^{k}=-u^{\prime} n_{k}, \quad t \Gamma_{i i}^{i}=+u^{\prime} n_{i}$,

$t \Gamma_{i 0}^{i}=-\varrho u^{\prime}, \quad t \Gamma_{i k}^{i}=+u^{\prime} n_{k}$.

Durch Substitution in (3.2) ergibt sich aus (3.4) zunächst

$$
A=3 t^{2} \partial_{0}\left(\frac{\varrho u^{\prime}}{t}\right)-3 \varrho^{2} u^{\prime},
$$

also

$$
A=-3 \varrho^{2}\left(u^{\prime \prime}+\frac{2}{\varrho} u^{\prime}+u^{\prime 2}\right) .
$$

Entsprechend ist

$$
\begin{aligned}
B n_{1}= & -t^{2} \partial_{1}\left(\frac{\varrho u^{\prime}}{t}\right)+t^{2} \partial_{1}\left(\frac{3 \varrho u^{\prime}}{t}\right) \\
& -3 \varrho u^{\prime} n_{1}+3 \varrho u^{\prime 2} n_{1},
\end{aligned}
$$

woraus

$$
B=2 \varrho\left(u^{\prime \prime}+\frac{1}{\varrho} u^{\prime}\right)
$$

hervorgeht. $D$ folgt aus

$D n_{1} n_{2}=t\left\{\partial_{1}\left(u^{\prime} n_{2}\right)+\partial_{2}\left(u^{\prime} n_{1}\right)-\partial_{2}\left(3 u^{\prime} n_{1}\right)\right.$

$$
\left.+\left(3 u^{\prime 2}+3 u^{\prime 2}-3 u^{\prime 2}-2 u^{\prime 2}\right) n_{1} n_{2}\right\},
$$

wonach

$$
D=-u^{\prime \prime}+\frac{1}{\varrho} u^{\prime}+u^{\prime 2}
$$

ist. Der letzte Beitrag berechnet sich wie folgt:

$$
\begin{aligned}
C+D n_{1}^{2}= & +t^{2} \partial_{0}\left(-\frac{\varrho f^{2} u^{\prime}}{t}\right)+t \partial_{1}\left(u^{\prime} n_{1}\right) \\
& -t \partial_{2}\left(u^{\prime} n_{2}\right)-t \partial_{3}\left(u^{\prime} n_{3}\right)-3 t \partial_{1}\left(u^{\prime} n_{1}\right) \\
& +3 Q^{2} f^{2} u^{\prime 2}+3 u^{\prime 2}\left(n_{1}^{2}-n_{2}^{2}-n_{3}^{2}\right) \\
& -2 \varrho^{2} f^{2} u^{\prime 2}-3 u^{\prime 2} n_{1}^{2}+2 u^{\prime 2}\left(n_{2}^{2}+n_{3}^{2}\right) .
\end{aligned}
$$

Das ist gleich

$$
\begin{array}{r}
\varrho^{2} f^{2}\left(u^{\prime \prime}+\frac{2}{\varrho} u^{\prime}+\right. \\
\left.+3 u^{\prime 2}\right)-\left(u^{\prime \prime}+\frac{3}{\varrho} u^{\prime}+u^{\prime 2}\right) \\
+\left(-u^{\prime \prime}+\frac{1}{\varrho} u^{\prime}+u^{\prime 2}\right) n_{1}^{2} .
\end{array}
$$

Der dritte Term liefert wiederum $D$. Somit ist

$$
C=Q^{2} f^{2}\left(u^{\prime \prime}+\frac{2}{\varrho} u^{\prime}+3 u^{\prime 2}\right)-\left(u^{\prime}+\frac{3}{\varrho} u^{\prime}+u^{\prime 2}\right) .
$$


Aus (3.1) erhält man mittels (3.4) und (3.6) für die Komponenten von $T_{\mu v}$ der Reihe nach

$$
\begin{aligned}
\varkappa t^{2} T_{00} & =+\frac{1}{2 f^{2}}\left(f^{2} A+3 C+D\right) \\
& =-\frac{2}{f^{2}}\left(u^{\prime \prime}+\frac{2}{\varrho} u^{\prime}+u^{\prime 2}\right)+3 \varrho^{2} u^{\prime 2}, \\
\varkappa t^{2} T_{0 i} & =+B n_{i}=2 \varrho\left(u^{\prime \prime}+\frac{1}{\varrho} u^{\prime}\right) n_{i}, \\
\varkappa t^{2} T_{i k} & =+\frac{1}{2}\left(f^{2} A-C-D\right) \delta_{i k}+D n_{i} n_{k} \\
& =-2 Q^{2} f^{2}\left(u^{\prime \prime}+\frac{2}{\varrho} u^{\prime}+\frac{2}{3} u^{\prime 2}\right) \delta_{i k} \\
& +\left(u^{\prime \prime}+\frac{1}{\varrho} u^{\prime}\right) \delta_{i k}-\left(u^{\prime \prime}-\frac{1}{\varrho} u^{\prime}-u^{\prime 2}\right) n_{i} n_{k} .
\end{aligned}
$$

Nach dem Modell einer sich gleichmäßig ausdehnenden Welt bewegen sich die Materiepunkte auf radialen Bahnen mit der Geschwindigkeit $\imath=r / t$ (betr. Kovarianz vgl. Gl. $(5.1-5)$ ). Danach ist die Beschleunigung gleich

$$
\frac{\mathrm{d} v}{\mathrm{~d} t}=\frac{v}{t}-\frac{r}{t^{2}}=0
$$

Die Massenelemente dürfen die durch das Modell vorgeschriebene Bahn nicht verlassen. Darum müssen die radialen Spannungen gleich 0 sein. Aus

$$
T_{i k} n_{k}=0
$$

erhält man mittels (3.12) die Differentialgleichung

$$
f^{2}=e^{2 u}=\frac{\frac{1}{\varrho} u^{\prime}+\frac{1}{2} u^{\prime 2}}{\varrho^{2}\left(u^{\prime \prime}+\frac{2}{\varrho} u^{\prime}+\frac{3}{\varrho} u^{\prime 2}\right)}
$$

In der Umgebung von $\varrho=0$ kann sich die Gravitation noch nicht auswirken. Darum ist $f(0)=1, u(0)=0$, und die quadratischen Terme in (3.13) sind im Limes in Strenge vernachlässigbar. Das führt zu der vereinfachten Differentialgleichung

$$
\frac{u^{\prime \prime}}{u^{\prime}}=-\frac{2}{\varrho}+\frac{1}{\varrho^{3}} .
$$

Daraus folgt mit $K$ als Integrationskonstante

$$
\begin{aligned}
& u^{\prime}=-\frac{K}{\varrho^{2}} e^{-1 / 2 \varrho^{2}}, \\
& u=-K \sqrt{\frac{\pi}{2}}\left[1-\Phi\left(\frac{\sqrt{1 / 2}}{\varrho}\right)\right],
\end{aligned}
$$

worin

$$
\Phi(x)=\frac{2}{\sqrt{\pi}} \int_{0}^{x} e^{-t^{2}} \mathrm{~d} t
$$

die Fehlerfunktion ist.

Da die Differentialgleichung für $\varrho>0$ regulär ist, und da an Stellen $\varrho>0$, an denen $u^{\prime}(\varrho)=0$ wäre, auch $u^{\prime \prime}$ und sämtliche höheren Ableitungen verschwinden würden, muß $u(\varrho)$ außer in $\varrho=0$ überall monoton fallend sein und kann daher asymptotisch negativ unendlich werden, so $\operatorname{da} \beta f=e^{u}$ asymptotisch verschwindet. Aus der zweiten Randbedingung

$$
f(\varrho) \rightarrow 0 \text { für } \varrho \rightarrow \infty
$$

folgt

$\frac{1}{\varrho} u^{\prime}+\frac{1}{2} u^{\prime 2}=0, \quad u^{\prime}=-\frac{2}{\varrho}, \quad u=-2 \ln \frac{\varrho}{a}$,

woraus

$$
f \rightarrow \frac{a^{2}}{Q^{2}}, \quad \sqrt{g}=f^{3} \rightarrow \frac{a^{6}}{Q^{6}}
$$

hervorgeht. Somit sind das Volumen $\tau$ und der Kugelradius $R$ gemäß

$$
\tau=\int \sqrt{g} \mathrm{~d} \tau, \quad R=\int \sqrt{g_{r r}} \mathrm{~d} r
$$

endlich. Klarerweise liefert die Integrationskonstante $a$ die Grenze $\varrho=a$, unterhalb $\operatorname{der} f=1$ und oberhalb der $f=a^{2} / Q^{2}$ nullte Näherung ist. Die Konstanten $K$ und $a$ werden nach vollständiger Integration durch Verknüpfung der beiden Enden von $f(\varrho)$ für $\varrho \rightarrow 0$ bzw. $\varrho \rightarrow \infty$ bestimmt sein. Man muß nämlich dafür sorgen, daß $u$ und $u^{\prime}$ überall stetig sind.

Damit ist die Existenz einer Metrik gesichert, die sich in der Nähe des Zentrums Minkowskisch verhält, und die einen Raum mit endlichem Volumen und eine Kugel mit endlichem Radius liefert. Wegen $f \rightarrow a^{4} / Q^{4}$ schrumpft der Raum im Unendlichen sozusagen in sich zusammen. Für die Berechnung des Volumens und verwandter Integrale muß man die Funktion $f(\varrho)$ genau kennen. Doch brauchen wir hier nur das Verhalten von $f(\varrho)$ bei $\varrho=0$ und für $\varrho \rightarrow \infty$. 
Im übrigen gibt es eine gewisse Verwandtschaft der Raum-Zeit zu derjenigen der Friedman-Welt. Transformiert man nämlich $\varrho \rightarrow \infty$ etwa mittels $\varrho^{\prime}=\varrho /(\varrho+1)$ auf $\varrho^{\prime}=1$, so verschwinden sämtliche Abstände auf der Kugelfläche $\varrho^{\prime}=1$. Es gibt sich also eine geschlossene Welt. Wegen des endlichen Volumens ist eine solche Transformation nicht abwegig. Ohne die Transformation bleibt es richtig, daß die transversalen Abstände mit $\varrho \rightarrow \infty$ verschwinden, so daß $u(\varrho)$ in jedem Falle eine geschlossene Welt darstellt.

\section{$\S 4$. Erhaltungsflächen}

Die Einsteinschen Feldgleichungen in (3.1) sind so bestimmt, daß die kovariante Energie-Impulsgleichung

$$
\nabla_{v} \mathfrak{T}^{\mu v}=\partial_{v} \mathfrak{I}^{\mu v}+\Gamma_{\varrho \sigma}^{\mu} \mathfrak{I}^{\varrho \sigma}=0
$$

gilt. Sie liefert wegen der $\Gamma$-Terme nicht ohne weiteres Erhaltungssätze. Da es auch in der Allgemeinen Relativitätstheorie kein Perpetuum mobile gibt, sind ergänzende Betrachtungen nötig (vgl. hierzu [15]).

Hier gehen wir davon aus, daß in der speziellen Relativitätstheorie Energie- und Impulserhaltung gelten, obwohl speziell relativistische Gleichungen auch allgemein kovariant geschrieben werden können, wobei der Energie-Impulssatz ebenfalls in der Form (4.1) erscheint.

In diesem Falle gibt es klarerweise Flächen $S(x)=$ const., z. B. die Minkowskischen Koordinatenebenen, relativ zu denen die Gleichung

$$
\nabla_{v}\left(\partial_{\mu} S \cdot \mathfrak{T}^{\mu v}\right)=\partial_{v}\left(\partial_{\mu} S \cdot \mathfrak{I}^{\mu v}\right)=0
$$

gilt. Das erste Gleichungszeichen ergibt sich, weil unter der Ableitung eine Vektordichte steht, für welche die kovariante Ableitung mit der differentiellen übereinstimmt. Das zweite besagt, daß es spezielle Flächen $S(x)=$ const. gibt, relativ zu denen die Erhaltungssätze

$$
P_{S}^{0}=\int \partial_{\mu} S \cdot \mathfrak{I}^{\mu 0} \mathrm{~d} \tau=\text { const }
$$

gelten. Wir sprechen darum von Erhaltungsflächen.

Integrabilität vorausgesetzt steht nichts im Wege, mittels (4.2) auch in der Allgemeinen Relativitätstheorie nach Erhaltungsflächen zu suchen. Insbesondere kann man nicht einwenden, in dieser Theorie könne es keine ausgezeichneten Flächen geben. Denn sie sind nach (4.2) allgemein kovariant definiert. Tatsächlich sind die Erhaltungsflächen auf das durch $\mathfrak{I}^{\mu v}$ definierte Objekt bezogen, hier also auf das kosmologische Modell.

Das erinnert an die Basisidee von Mach [16]. Er ist von Zweifeln an der Newtonschen Deutung des Eimerversuchs [17] ausgegangen und hat behauptet, bei der Wölbung der Wasseroberfläche in einem rotierenden Eimer handele es sich um eine Folge der Relativbewegung zum Fixsternhimmel. Diese Bemerkung hat zwar Einstein auf seinen Weg geführt, doch sind alle Bemühungen gescheitert, das Machsche Prinzip allgemein relativistisch zu begründen.

Wir meinen, Gl. (4.2) zeige, warum jene Bemühungen keinen Erfolg haben konnten. Es gibt nämlich im allgemeinen keine Minkowski-Koordinaten. Doch macht (4.2) zugleich deutlich, daß Mach mit seiner Idee das Richtige getroffen hat. Die Erhaltungsflächen sind nämlich durch die globale Energie-Impulsverteilung, also wie bei Mach kosmologisch bestimmt.

Man begegnet auch Zweifeln an der Integrabilität von (4.2). Sie werden durch das Verhalten lokal definierter Systeme genährt. Doch kann das kaum allgemein richtig sein. Beispielsweise hat man keine Zweifel bei der mathematisch analog definierten Wellengleichung für skalare Funktionen $\Phi(x)$ in der Riemann-Minkowskischen Raum-Zeit

$$
\partial_{\mu}\left(\mathfrak{g}^{\mu v} \partial_{v} \Phi\right)=0 .
$$

Mindestens in endlichen Umgebungen regulärer Punkte wird es Integrale und damit auch Erhaltungsflächen geben. Singularitäten wären ein Anzeichen, daß $g_{\mu v}$ nur innerhalb gewisser Grenzen physikalisch sinnvoll definiert ist. (Physikalisch könnte das z. B. davon herrühren, daß es keine in Strenge abgeschlossenen und räumlich begrenzten Systeme gibt, daß sie vielmehr alle in den globalen Untergrund eingebettet sein müssen. Wir lassen diese Frage offen.) Jedenfalls kann man schon bei der FriedmanWelt leicht nachrechnen, daß die Gleichung für Erhaltungsflächen global integrabel ist.

Die Existenz von Erhaltungsflächen hat für die Welt im Werden eine wichtige Konsequenz. In der Minkowskischen Raum-Zeit ist $S=t$ eine spezielle Erhaltungsfläche, nämlich die für die Energie. Hier muß sich diese Gleichung z. B. in $Q=0$ einstellen. Darum ist die Dimension von $S$ gleich $s$ bzw. $m$ im Falle $c=1$. Somit ist $\partial_{\mu} S$ in (4.3) dimensionslos. In 
denselben Einheiten haben $G$ und $\mathfrak{I}^{\mu v}$ die Dimensionen $\mathrm{kg}^{-1} \mathrm{~m}$ bzw. $\mathrm{kg} \mathrm{m}^{-3}$. Die Dimension des Integrals ist also gleich $\mathrm{kg}$. Das Integral muß durch $G$ ausdrückbar sein. Folglich ist $P_{S}^{0} \sim t / G$. Das ist nur dann konstant, wenn der Faktor verschwindet. Danach ist die Gesamtenergie

$$
W_{\text {tot }}=0 .
$$

Daraus folgt, daß der Integrand neben positiven auch negative Beiträge enthalten muß. Diese rühren schon nach dem Newtonschen Gesetz vom Gravitationsfeld her. Da auf der rechten Seite der Feldgleichungen (3.1) die Quellen des Gravitationsfeldes stehen, muß auch das Gravitationsfeld gravitierend wirken. Es muß also bei der Gravitation Feld-Feldwechselwirkungen geben. Bisher hat man angenommen, wohl ohne mit vorangegangenen Voraussetzungen in Konflikt zu geraten, da $\beta \mathfrak{T}^{\mu \nu}$ allein durch Massen und elektromagnetische, sowie durch die mit Elementarteilchen verbundenen Felder bestimmt sei. Hier besteht nicht mehr die Freiheit der Wahl. Aus dem Linienelement in (2.2) folgt, daß das Gravitationsfeld wie alle anderen Felder Gravitationsfelder erzeugt. Darum kommt auch auf der rechten Seite von (3.1) das Gravitationsfeld vor.

Das ist nicht so befremdlich, wie es auf den ersten Blick scheinen mag. Bei Jordans Versuch [18], die bereits oben erwähnte Idee von Dirac allgemein relativistisch zu formulieren, hat er ausdrücklich die Voraussetzung eingeführt, daß die Gesamtenergie einschließlich der des Gravitationsfeldes gleich 0 sei. Es ist höchst befriedigend, daß sich hier die Jordansche Forderung als Folgesatz einstellt, ohne daß man die Einsteinsche Feldgleichungen abändern muß. Ähnlich wie oben die Diracsche Vermutung ergibt sich hier die Jordansche Forderung als Folge des Ansatzes von $\mathrm{d} s^{2}$ in (2.2) von selbst. Außerdem muß man bedenken, daß die ständige Erzeugung von Materie mit der Energieerhaltung nur dann verträglich ist, wenn neu erzeugte Energie zugleich durch die mitentstehende Gravitationsenergie kompensiert wird.

Danach zerfällt der Energie-Impulstensor gemäß

$$
\mathfrak{I}^{\mu v}=\mathfrak{I}_{\text {mat }}^{\mu v}+\mathfrak{T}_{\text {grav }}^{\mu v}
$$

in einen Beitrag, den man kurz den materiellen nennen kann, und in einen der Gravitation. Dabei sind die Erhaltungsflächen klarerweise auf den Gesamttensor bezogen. Sie liefern analog zu (4.3) auch den Beitrag der Materie zu den Erhaltungsgrößen

$$
P_{\text {mat }}^{0}=\int \partial_{\mu} S \cdot \boldsymbol{T}_{\text {mat }}^{\mu 0} \mathrm{~d} \tau .
$$

Er ist klarerweise nicht mehr konstant und wächst aus Dimensionsgründen proportional zur Zeit.

$\mathfrak{I}_{\text {mat }}^{\mu v}$ muß aus den jeweiligen zuständigen, hier z. B. aus den hydrodynamischen Gleichungen, in der durch $\mathrm{d} s^{2}$ definierten Riemann-Minkowskischen Raum-Zeit berechnet werden. Der Gravitationsanteil ergibt sich dann als Differenz

$$
\mathfrak{I}_{\text {grav }}^{\mu v}=\mathfrak{I}^{\mu v}-\mathfrak{I}_{\text {mat }}^{\mu v} .
$$

Sofern es gelingt, $\mathfrak{I}_{\text {mat }}^{\mu v}$ eindeutig zu bestimmen, was eine passende Wahl von $\mathrm{d} s^{2}$ voraussetzt, haben wir eine strenge Lösung. Natürlich erhält man auf diese Weise nur spezielle Lösungen. Die allgemeinste Form eines Linienelementes von obiger Art wäre

$$
\mathrm{d} s^{2}=\mathrm{d} t^{2}-g_{i k}(\varrho) \mathrm{d} x^{i} \mathrm{~d} x^{k}, \quad \varrho=r / t .
$$

Auch hiernach ist im Ruhesystem $\mathrm{d} s=\mathrm{d} t$.

\section{§ 5. Energie-Impulstensor einer Gaskugel}

Die Metrik in (2.2) rühre von einem Gas her, welches die Welt im Werden erfüllt und relativ zu der sich ausdehnenden Kugel ruht. In diesem Falle ist die lokale Geschwindigkeit des Gases

$$
\boldsymbol{v}=\varrho \boldsymbol{n} .
$$

Wegen $g_{\mu v} u^{\mu} u^{v}=-1$ folgt daraus für die Vierergeschwindigkeit

$$
\left(u^{0} ; \boldsymbol{u}\right)=\frac{1}{\sqrt{1-f^{2} \varrho^{2}}}(1, \varrho \boldsymbol{n}) .
$$

Da die Gesamtmasse proportional zu $t$ wachsen muß, ist die Massendichte gleich

$$
\hat{\mu}=\frac{1}{t^{2}} \mu(\varrho)=\frac{1}{t^{2}} \mu_{0}(\varrho) f(\varrho)^{3} .
$$

Darin ist $\mu_{0}$ ein Skalar und $\mu$ wegen $f^{3}=\sqrt{g}$, $g=-\operatorname{det}\left(g_{\mu \nu}\right)$ eine skalare Dichte. Die Dimensionen von $\hat{\mu}, \mu$ und $\mu_{0}$ sind wegen $c=1 \mathrm{~kg} \mathrm{~m}^{-3}$, $\mathrm{kg} \mathrm{m}^{-1}$ und $\mathrm{kg} \mathrm{m}^{-1}$. Die kovarianten Ableitungen lauten

$$
\nabla_{\mu}\left(\frac{\mu}{t^{2}}\right)=\partial_{\mu}\left(\frac{\mu}{t^{2}}\right)-\frac{1}{t^{2}} \Gamma_{i \mu}^{\lambda} \mu .
$$


Darin ist

$\partial_{0}\left(\frac{\mu}{t^{2}}\right)=-\frac{1}{t^{3}}\left(2 \mu+\varrho \mu^{\prime}\right), \quad \nabla\left(\frac{\mu}{t^{2}}\right)=\frac{1}{t^{3}} \mu^{\prime} \boldsymbol{n}$.

Die Stromdichte ist gleich

$$
\mathfrak{J}^{\mu}=\frac{1}{t^{2}} \mu u^{\mu} \text {. }
$$

Obige Gleichungen folgen unmittelbar aus dem Modell. Von (5.1) haben wir schon früher Gebrauch gemacht.

$\mathfrak{J}^{\mu}$ kann die Kontinuitätsgleichung nicht befriedigen, weil die Masse wächst. Es muß also Quellen für die zunehmende Masse geben, und damit einen Quellenterm auf der rechten Seite der Kontinuitätsgleichung. Es entbehrt nicht eines gewissen Reizes, die Quellen ins Zentrum zu setzen, so als gäbe es dort einen deus ex machina, der ständig Materie nachschaufelt und dabei für das Geschehen in der Welt sorgt.

Doch sind wir in unseren Annahmen nicht frei. Bedenkt man nämlich, daß in der Quantenphysik die Erzeugung und Vernichtung von Teilchen in Teilchen verankert ist, so wird man die Quellen der kosmischen Materieerzeugung in der kosmischen Materie verankern müssen und darum mit der Gleichung

$$
\partial_{\mu} \mathfrak{J}^{\mu}=\frac{1}{t^{3}} q
$$

beginnen. Daraus folgt mittels des Gaußschen Satzes, weil $\mathfrak{J}^{\mu}$ wegen (3.15) asymptotisch hinreichend rasch verschwindet,

$$
\frac{\mathrm{d}}{\mathrm{d} t}\left(4 \pi t \int \frac{\mu \varrho^{2} \mathrm{~d} \varrho}{\sqrt{1-f^{2} \varrho^{2}}}\right)=4 \pi \int q \varrho^{2} \mathrm{~d} \varrho .
$$

Danach ist die Erzeugungsrate für die Gesamtmasse $M$ gleich

$$
\frac{\mathrm{d} M}{\mathrm{~d} t}=4 \pi \int \frac{\mu Q^{2} \mathrm{~d} \varrho}{\sqrt{1-f^{2} Q^{2}}}=4 \pi \int q \varrho^{2} \mathrm{~d} Q .
$$

Die Massenerzeugung ist Sache des Gravitationsfeldes. Darum ist es gleichgültig, von welcher Art von Teilchen die Erzeugung herrührt. Alle Elemente der Quelle müssen in gleicher Weise wirken, so daß nicht nur die Integrale, sondern auch die Integranden übereinstimmen müssen. Somit ist die Quelldichte

$$
q=\frac{\mu}{\sqrt{1-f^{2} Q^{2}}} .
$$

Damit lautet die erweiterte Kontinuitätsgleichung

$$
\partial_{\mu}\left(\frac{\mu}{t^{2}} u^{\mu}\right)=\frac{1}{t^{3}} \frac{\mu}{\sqrt{1-f^{2} Q^{2}}} .
$$

Durch Ausrechnung erhält man nach Multiplikation mit $t^{3}$

$-\frac{2 \mu}{\sqrt{ }}-\varrho\left(\frac{\mu}{\sqrt{ }}\right)^{\prime}+\frac{2}{\varrho} \frac{\varrho \mu}{\sqrt{ }}+\left(\frac{\varrho \mu}{\sqrt{ }}\right)^{\prime}=\frac{\mu}{\sqrt{ }}$.

Das ist offensichtlich eine Identität, so daß jede Massendichte $\mu(\varrho)$ zulässig ist.

Ähnliches könnte man für ein Gas in einer Glaskugel sagen, solange man noch keine dynamischen Gesetze beachtet. Diese führen in der Glaskugel am Ende zu einem Gleichgewicht, in dem die Massendichte konstant ist. Vielleicht ergibt sich hier Vergleichbares. Einstweilen verzichten wir darauf, solche Gesetze zu formulieren. Gestützt auf astrophysikalische Erfahrungen nehmen wir an, daß

$$
\mu_{0}=\text { const }
$$

ist. Da das Volumenelement $\mathrm{d} \tau^{\prime}=\sqrt{g} \mathrm{~d} \tau=f^{3} \mathrm{~d} \tau$ ist, erhält man nämlich für die Dichte

$$
\frac{\mathrm{d} m}{\mathrm{~d} \tau^{\prime}}=t \frac{\mu_{0} f^{3} \mathrm{~d} \tau}{f^{3} \mathrm{~d} \tau}=t \mu_{0},
$$

so daß (5.10) mit $\mu_{0}$ statt $\mu$ angemessen erscheint.

In unserem rohen Bild, in dem die Sterne Gasmoleküle sind, liegt die freie Weglänge um viele Größenordnungen über dem Weltradius, so daß man einen Gasdruck nicht zu berücksichtigen braucht. Wir haben also nur mit dem kinetischen Beitrag zum Energie-Impulstensor zu rechnen, also mit

$$
\mathfrak{I}_{\mathrm{mat}}^{\mu v}=\frac{1}{t^{2}} \mu u^{\mu} u^{v} .
$$

Mit den Erhaltungsflächen $S$ erhält man auf der linken Seite der Gleichungen für Energie und Impuls

$$
\begin{aligned}
& \partial_{v}\left(\frac{\mu}{t^{2}} u^{v}\left(u^{\mu} \partial_{\mu}\right) S\right) \\
& \quad=\frac{1}{t^{3}} \frac{\mu}{\sqrt{1-f^{2} Q^{2}}}\left(u^{\mu} \partial_{\mu}\right) S+\frac{\mu}{t^{2}}\left(u^{v} \partial_{v}\right)\left(u^{\mu} \partial_{\mu}\right) S,
\end{aligned}
$$

letzteres nach (5.9). Auf der rechten Seite steht die Quelldichte für Energie und Impuls $Q_{S} / t^{3}$.

An dieser Stelle müssen wir die Differentialgleichung (4.2) für die Erhaltungsflächen $S$ explizite 
betrachten. Die Gleichgewichtsbedingung $T_{i k} n_{k}=0$ vor (3.13) ergibt nach (3.12)

$$
f^{2} A=C-D \text {. }
$$

Danach ist

$$
\varkappa t^{2} T_{i k}=+D\left(n_{i} n_{k}-\delta_{i k}\right),
$$

und den Energie-Impulstensor kann man in der Form

$\left(\varkappa f t^{2} \mathfrak{T}^{\mu v}\right)$

$$
=\left(\begin{array}{llll}
2 f^{2} C, & -f^{2} B n_{1}, & -f^{2} B n_{2}, & -f^{2} B n_{3} \\
-f B n_{1}, & D\left(n_{1}^{2}-1\right), & D n_{1} n_{2}, & D n_{1} n_{3} \\
-f B n_{2}, & D n_{2} n_{1}, & D\left(n_{2}^{2}-1\right), & D n_{2} n_{3} \\
-f B n_{3}, & D n_{3} n_{1}, & D n_{3} n_{2}, & D\left(n_{3}^{2}-1\right)
\end{array}\right) .
$$

Damit erhält man aus (4.2)

$$
\begin{aligned}
& \partial_{0}\left(\frac{2 f C}{t^{2}} \partial_{0} S-\frac{f B}{t^{2}} \boldsymbol{n} \cdot \nabla S\right) \\
& +\operatorname{div}\left(-\frac{f B}{t^{2}} \boldsymbol{n} \partial_{0} S+\frac{D}{f t^{2}}(\boldsymbol{n} \boldsymbol{n} \cdot \nabla S-\nabla S)\right)=0 .
\end{aligned}
$$

Wir betrachten speziell die Erhaltungsflächen der Energie. In Hinblick auf $S=t$ im Minkowski-Fall gehen wir hier von

$$
\begin{aligned}
& S=t \cdot g(\varrho), \quad \partial_{0} S=g-\varrho g^{\prime}, \\
& \nabla S=\boldsymbol{n} g^{\prime}, \quad g(0)=1, \quad g^{\prime}(0)=0
\end{aligned}
$$

aus. Das führt zu einer Differentialgleichung für $g$, die gemäß

$$
\begin{aligned}
\partial_{0}\left(\frac{2 f C}{t^{2}}\right. & \left.\left(g-\varrho g^{\prime}\right)-\frac{f B}{t^{2}} g^{\prime}\right) \\
& +\operatorname{div}\left(-\frac{f B}{t^{2}}\left(g-\varrho g^{\prime}\right) n\right)=0
\end{aligned}
$$

für $\varrho>0$ regulär ist.

Setzt man in $B$ und $C$ die asymptotischen Werte $u^{\prime}=-2 / \varrho, u^{\prime \prime}=2 / \varrho^{2}$ und $f=a^{2} / \varrho^{2}$ ein, so folgt

$$
B=0, \quad C=10 f^{2}=10 \frac{a^{4}}{\varrho^{2}} .
$$

Damit erhält man aus (5.15)

$$
\begin{aligned}
& \partial_{0}\left(\frac{20 f^{2}}{t^{2}}\left(g-\varrho g^{\prime}\right)\right) \\
& \quad=-\frac{1}{t^{3}}\left\{40 f^{2}\left(g-\varrho g^{\prime}\right)+20 \varrho\left(f^{2}\left(g-\varrho g^{\prime}\right)\right)^{\prime}\right\}=0 .
\end{aligned}
$$

Eine erste Integration führt zu

$$
f^{2}\left(g-\varrho g^{\prime}\right)=\frac{K}{\varrho^{2}},
$$

woraus

$$
g-\varrho g^{\prime}=\frac{K}{a^{4}} \varrho^{2}
$$

und

$$
g=-\frac{K}{a^{4}} \varrho^{2}+K^{\prime} \varrho
$$

hervorgehen. Die Konstanten $K$ und $K^{\prime}$ ergeben sich durch Anschluß an die Anfangswerte $g(0)=1$ und $g^{\prime}(0)=0$. Darauf brauchen wir hier nicht einzugehen.

Substitution von (5.14) in (5.12) ergibt

$$
\begin{aligned}
& \text { und } \\
& \begin{aligned}
\left(u^{v} \partial_{v}\right)\left(u^{\mu} \partial_{\mu} S\right) & =\frac{1}{\sqrt{ }}\left(\partial_{0}+p \partial_{v}\right) \frac{g}{\sqrt{ }} \\
= & \frac{1}{t}\left\{-\frac{\varrho}{\sqrt{ }}\left(\frac{g}{\sqrt{ }}\right)^{\prime}+\frac{\varrho}{\sqrt{ }}\left(\frac{g}{\sqrt{ }}\right)^{\prime}\right\}=0 .
\end{aligned}
\end{aligned}
$$$$
\begin{aligned}
\left(u^{\mu} \partial_{\mu} S\right) & =\frac{1}{\sqrt{ }} \partial_{0} S+\frac{\varrho}{\sqrt{ }} \partial_{v} S \\
& =\frac{g-\varrho g^{\prime}}{\sqrt{ }}+\frac{\varrho g^{\prime}}{\sqrt{ }}=\frac{g}{\sqrt{ }}
\end{aligned}
$$

Danach trägt nur der erste Summand im mittleren Ausdruck von (5.12) zur Quelldichte der Energie $Q / t^{3}$ bei. Mittels (5.14) folgt daraus

$$
Q=\frac{\mu g}{1-f^{2} Q^{2}} .
$$

Somit ist die Erzeugungsrate der Energie gleich

$$
\frac{\mathrm{d} W_{\mathrm{mat}}}{\mathrm{d} t}=4 \pi \int \frac{\mu g \varrho^{2}}{1-f^{2} \varrho^{2}} \mathrm{~d} \varrho .
$$

In Verbindung mit (5.7) erhält man als Erzeugungsrate der kinetischen Energie

$$
\begin{aligned}
& \frac{\mathrm{d}}{\mathrm{d} t}\left(W_{\mathrm{mat}}-M\right) \\
& \quad=4 \pi \int \frac{\mu}{\sqrt{1-f^{2} \varrho^{2}}}\left(\frac{g}{\sqrt{1-f^{2} \varrho^{2}}}-1\right) \varrho^{2} \mathrm{~d} \varrho .
\end{aligned}
$$

Nach (5.18) und (5.7) liefern $W_{\text {mat }}$ und $M$ die Energie der Materie bzw. ihre Menge, nämlich die Summe der Ruhmassen aller Sterne. Die Konvergenz wird durch den Faktor $f^{3}$ in $\mu$ garantiert. 
Wir begnügen uns mit diesen Existenzbeweisen. Zur Berechnung von Zahlenfaktoren wie $\gamma$ in $M=\gamma t / G c^{3}$ müßte man $f$ und $g$ explizite ausrechnen. Vielleicht muß man vorher das noch recht grobe Modell aufgrund astrophysikalischer Erfahrungen verfeinern.

Die Welt im Werden ist eine in sich konsistente Lösung der Einsteinschen Feldgleichungen. Sie konkurriert mit der Friedman-Welt und unterscheidet sich von dieser in den ersten drei Minuten erheblich. Die Entscheidung zwischen beiden Modellen wird vielleicht erst quantenphysikalisch möglich sein. Doch gibt es eine Reihe von Ergebnissen, die schon jetzt positiv zu Buche schlagen:

1. Der Weltradius kommt nicht zweimal ins Spiel.

2. Die natürlichen Randbedingungen ergeben eine endliche Welt.

3. Wie Dirac vermutet hat, sind Masse und Weltalter proportional, hier ohne Abänderung der Feldgleichungen.

4. Die nach dem Hubbleschen Gesetz zu erwartende Ausdehnung steckt hier als phänomenologische Basis bereits im Ansatz des Modells der Gaskugel

5. Vom Beitrag der noch nicht gut bekannten Dunkelmaterie wird zwar der Weltradius abhängen, doch sind Welten mit großer und kleiner Massendichte nicht mehr qualitativ verschieden.

6. Die Gesamtenergie ist null. Masse und Energie der Materie wachsen proportional zur Zeit. Der Energiesatz ist bei Materieerzeugung klarerweise nur erfüllt, wenn die Zunahme der Materieenergie durch eine gleichgroße Abnahme der Gravitationsenergie kompensiert wird (vgl. dazu aber Thirring [19]).

Für kritische und die Klarheit fördernde Diskussionen habe ich den Herren Ehlers, Schmutzer und Thirring zu danken. Es versteht sich von selbst, daß eine einzige Arbeit noch nicht viele vorangegangene Arbeiten außer Kraft setzt, zumal das FriedmanModell ästhetisch sehr befriedigend ist. Doch geht

[1] E. Schmutzer, Relativistische Physik, Leipzig 1968; hier S. $852 \mathrm{ff}$. Wir zitieren möglichst aus diesem Buche. Jedes andere, ähnlich umfassende kann man zu Rate ziehen.

[2] Einstein, l.c. [1], S. $377 \mathrm{ff}$.

[3] I. Newton. Philosophiae Naturalis Principia Mathematica; Übersetzung ins Englische von A. Motte, 1729; Neuherausgabe von F. Cajori: Sir Isaac es nicht nur um eine neue strenge Lösung der Einsteinschen Feldgleichungen. Die Berufung auf Newtons heute nicht gerade beliebte Wissenschaftslehre ist zu gewichtig, weil sie in wunderbarer Weise die vielen Facetten der gegenwärtigen Physik als Einheit zu verstehen erlaubt. Den Vorrang der Phänomene vor Axiomen und daraus folgenden Theorien habe ich kürzlich privatim an einem drastischen Beispiel in Versen erläutert. Vielleicht ist es nicht abwegig, sie hier zum Abdruck zu bringen.

Viertausend Jahre ungefähr,

Sagen die Leute, ist es her,

Seit ein Hethiter Eisen entdeckt,

Seit er gefunden, wie man es macht.

Handel zu treiben, hat er bezweckt.

Warum es geht, hat kaum er bedacht.

Immer noch gilt, wie jedermann weiß:

„Kohle mit Erz vermeng' und mach' heiß!“

Alchemisten und Chemisten haben manches dann erklärt.

Doch berichten uns Chronisten, vieles davon sei verkehrt.

Denn die Bilder, die wir machen, müssen dies und das erfassen.

Sie umschließen Siebensachen, ohne neue zuzulassen.

Darum wandeln Bilder stets sich, wenn Erkenntnis schreitet fort.

Nur Rezepte gelten ewig, jeder Zeit an jedem Ort.

Axiome, auf die wir beim Denken bauen,

Verdienen nach Newton nur dann Vertrauen,

Wenn und so lang sie in Fakten wurzeln.

Neue Entdeckungen lassen sie purzeln.

Denn Bilder sind unsre und niemals ganz treue.

Doch müssen beim Fortschritt sie alte und neue

Fakten umschließen. - Daraus erhellt:

Ewig ist nur die Ordnung der Welt.

Newton's Mathematical Principles; Univ. of Calif. Press, Berkeley 1962: hier: Scholium Generale, vorletzter Absatz, S. 547.

[4] F. Bopp, Newtons Wissenschaftslehre als Basis der Quantenphysik. Ann. Phys. 7. Folge, 42, 217 (1985).

[5] H. Hubble, 1.c. [1], S. 848.

[6] A. Einstein, 1.c. [1], S. 849.

[7] H. v. Seeliger, l.c. [1], S. 848, Fußnote. 
[8] A. Friedman, 1.c. [1], Fußnote.

[9] F. Bopp, Uber die Einheit der klassischen Physik. Sitz.-Ber. Bay. Akad. Wiss. 1983, S. 53 ff., S. 74.

[10] S. Weinberg, The First Three Minutes. Basic Books Inc., New York 1977.

[11] C. Schäfer und H. Dehnen, Astron. Astrophys. 54, 823 (1976).

[12] F. Bopp, Erzeugungs- und Vernichtungsoperatoren der Quantenphysik beschreiben lokale Prozesse. Z. Naturforsch. 39 a, 113 (1983).
[13] P. A. M. Dirac, 1.c. [1], S. 906, Fußnote.

[14] A. Einstein, l.c. [2].

[15] E. Schmutzer, l.c. [1], S. $874 \mathrm{ff}$.

[16] E. Mach, Die Mechanik in ihrer Entwicklung, 8. Aufl., F. A. Brockhaus, Leipzig 1921.

[17] I. Newton, 1.c. [3], erstes Scholium, Abs. 13, S. 10.

[18] P. Jordan, l.c. [1], S. 906, Fußnote.

[19] W. Thirring, Lehrbuch der Mathematischen Physik, Springer-Verlag, Wien 1978, Bd. II, 1. 3, 35. 